\title{
Translational and Rotational Diffusion of Spin Probes in Nylon Films
}

\author{
Kunihiro Hamada, Katsuhiro AMACHI, Koichiro YonetaKe, ${ }^{\dagger}$ \\ Toshiro IIJIMA, and Ralph MCGREGOR* \\ Department of Polymer Science, Tokyo Institute of Technology, \\ Ookayama, Meguro-ku, Tokyo 152, Japan \\ * Department of Textile Chemistry, North Carolina State University, \\ Raleigh, North Carolina 27695-8302, U.S.A.
}

(Received December 10, 1986)

\begin{abstract}
The translational diffusion of nitroxide spin probes in nylon films was investigated by means of a sublimative desorption method, and compared with their rotational diffusion as determined by an electron spin resonance (ESR) technique in our previous papers. The translational diffusion coefficients $D$ calculated using McBain's formula were dependent on the structures of the spin probes, and on the structures of the nylons. The Arrhenius plots of $D$ defined one distinct crossover point for each probe in the temperature range examined, which was quite consistent with that found in the Arrhenius plots of the rotational diffusion coefficients $R$. This suggests that the motional change of the nylon chains affects not only the translational motions of the probes but also their rotation. However, the activation energies for translational and rotational diffusion were very different; the activation energies for translation were larger below the crossover point than above it, whereas those for rotation behaved in the opposite manner. This discrepancy can perhaps be explained by the differences between the time-scales of the motions detected in the translational and rotational measurements. The results obtained here are compared with those for polyethylene, natural rubber, and polyisobutylene reported elsewhere.

KEY WORDS Spin Probe / Nitroxide / Diffusion / Nylon / Molecular Motion /
\end{abstract}

The relationship between translational and rotational diffusion is of interest in the fields of membrane science and dyeing chemistry. Knowledge of this relationship should provide useful information on the mechanism of transport.

We have investigated the rotational mobilities of spin probes in nylon films by means of an electron spin resonance (ESR) technique. $^{1-6}$ It was found that the rotational mobilities were strongly influenced not only by the structures of the spin probes and of the nylons, but also by the molecular interactions between the probe molecules and the polymer chains. In addition, the rotational motions of the probe molecules in the "high" temperature region were interpreted by taking into account their simultaneous translational diffusion. ${ }^{3}$ From this point of view, it is worthwhile to examine the translational diffusion of the spin probes by an independent method, for comparison with their rotational diffusion.

Some papers have reported on the relationships between translational and rotational diffusion of nitroxide spin probes in polymer matrices. ${ }^{7-11}$ Stryukov used a method of scan-

† Permanent address: Department of Polymer Chemistry, Faculty of Engineering, Yamagata University, Yonezawashi, Yamagata 992, Japan. 
ning thin layers to determine the translational diffusion coefficients $D$ of TEMPO in polyethylene. ${ }^{7} \mathrm{He}$ concluded that the ratio of $D$ to the rotational diffusion coefficient $R$ depended on temperature, and consequently is not determined solely by the effective dimensions of the probe molecule. On the other hand, Vasserman et al. reported that the ratio $D / R$ for the nitroxide radical is dependent on the structure and flexibility of the main polymer chain, on the basis of $D$ values determined by a desorption method. ${ }^{9}$

In the present study, a sublimative desorption method was used to determine the translational diffusion coefficients of two nitroxide spin probes in two nylon films, for comparison with their rotational motions as investigated in our previous papers. ${ }^{2,6}$ This desorption method has been widely used to investigate the progressive diffusion of dyes and their model compounds in polymer films such as poly(ethylene terephthalate), ${ }^{12}$ polypropylene, ${ }^{13}$ polystyrene, ${ }^{14}$ and so on. Here we discuss the relationships between the activation energies for translation and rotation, taking into consideration the structures of the probe molecules and of the nylons.

\section{EXPERIMENTAL}

\section{Samples}

The two nonionic spin probes, 4-((pphenylazobenzoyl)amino)-2,2,6,6-tetramethylpiperidine-1-oxy (SPI) and 2,2,6,6-tetramethylpiperidine-1-oxy (SPIV) were used.

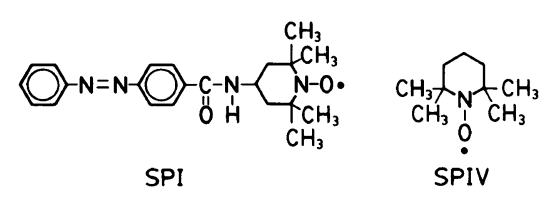

SPI was prepared as described in our previous paper." SPIV was purchased from Aldrich Co., and used without further purification. The two nylon films used here, biaxially drawn nylon 6 film (NY-6(2D)) and nylon 11 film drawn by inflation (NY-11), were kindly supplied by Unitika Co. and Daicell Co., respectively. The thicknesses of NY-6(2D) and NY-11 were 15 and $37 \mu \mathrm{m}$, respectively. All the films were pretreated in boiling aqueous ethanol for $3 \mathrm{~h}$, and in boiling water for $3 \mathrm{~h}$, to stabilize their physical structures and to remove their oligomers.

The spin probes were sorbed by the nylon films from aqueous solution at $343 \mathrm{~K}$ for 2 days. The amounts of the probes sorbed were determined to be in the range $(1-5) \times$ $10^{-5} \mathrm{~mol} \mathrm{~g}^{-1}$ of nylon by spectrophotometric measurements of the initial and final bath concentrations. After sorption, the films were rinsed with cold water, blotted with filter paper, and dried in a silica gel desiccator for a week.

\section{Sublimative Desorption Measurements}

The sublimative desorption apparatus shown in Figure 1 was used, and is similar to that used by Okajima et al. ${ }^{12}$ In this apparatus two sublimation tubes are available simultaneously. Strips measuring $1.0 \mathrm{~cm} \times 4.0 \mathrm{~cm}$ were cut out of the film containing the spin probe, placed in a sublimation tube, preheated for $20 \mathrm{~min}$, and then the tubes were evacuated to achieve a pressure less than $1 \times 10^{-3}$ Torr. After a desired time $t$, the vacuum was broken, and the spin probe remaining in the strips was extracted with $40 \mathrm{ml} 50 \%$ aqueous ethanol solution to measure its concentration spectrophotometrically. Here the wavelengths of maximum absorption for SPI and SPIV were 325 and $244 \mathrm{~nm}$, respectively.

Measurement of the amount of the spin probe remaining in the film $\left(M_{t}{ }^{\prime}\right)$ led to estimates of the amount $M_{t}$ desorbed during a time interval $t\left(M_{t}=M_{\infty}-M_{t}{ }^{\prime}\right)$, where $M_{\infty}$ is the value of $M_{t}$ for $t=\infty$. In the present study, the initial spin probe concentration in the film was used for $M_{\infty}$. 


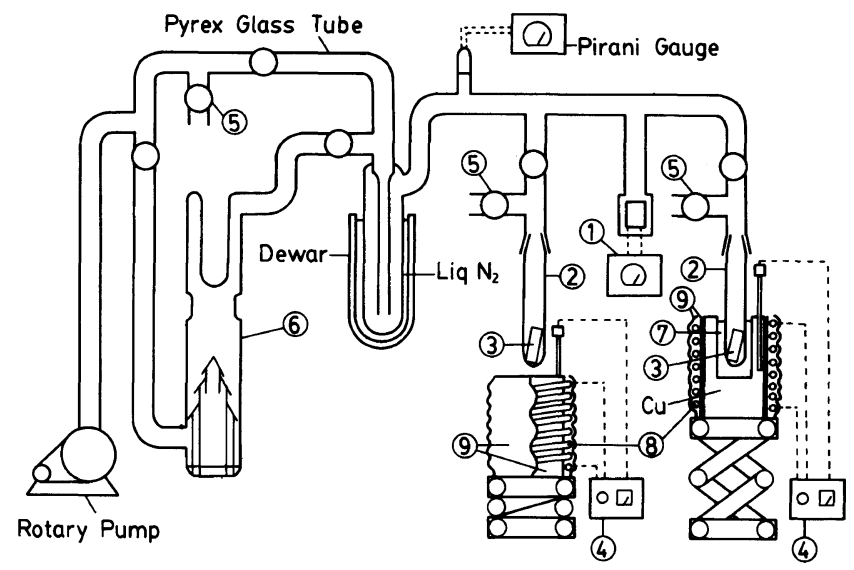

Figure 1. Schematic representation of the desorption apparatus. 1, ionization gauge; 2, sublimation tube; 3 , sample; 4 , heater controller; 5 , leak valve; 6 , diffusion pump; 7 , silicone oil; $8, \mathrm{Ni}-\mathrm{Cr}$ wire; 9 , asbestos.

\section{RESULTS AND DISCUSSION}

The reduced desorption curves of SPI and SPIV at various temperatures are shown in Figures 2 and 3. All the plots of $M_{t} / M_{\infty}$ against $t^{1 / 2}$ showed a good linearity in the range of $M_{t} / M_{\infty}<0.6$. From those straight lines, the translational diffusion coefficients $D$ of the spin probes in the nylon films were determined using the following equation: ${ }^{14}$

$$
\frac{M_{t}}{M_{\infty}}=\frac{4}{l}\left(\frac{D}{\pi}\right)^{1 / 2} t^{1 / 2}
$$

Where $l$ is the thickness of the film.

The straight lines for SPI did not pass through the origin, while those for SPIV did so within experimental error. This might be due to the larger molar volume of SPI and its stronger interaction with the nylon chains, as pointed out in our previous papers. ${ }^{2,3}$ These factors make it difficult for SPI to sublime out of the film and consequently to achieve the condition required for eq 1 , under which the surface probe concentration equals zero and the inner probe concentration is constant. On the other hand, SPIV with the smaller molar volume and the weaker interaction with the nylon chains ${ }^{6}$ is thought to achieve such a condition easily. The observations of Okajima

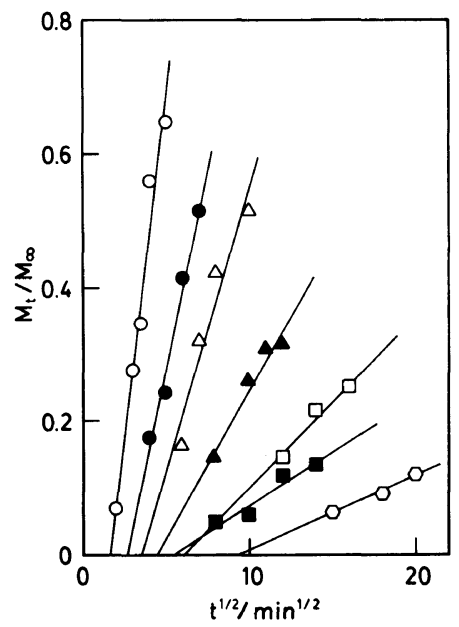

Figure 2. Reduced desorption curves for SPI in NY11. $\bigcirc, 431 \mathrm{~K}$;, $421 \mathrm{~K} ; \triangle, 411 \mathrm{~K} ; \Delta, 401 \mathrm{~K} ; \square, 396 \mathrm{~K}$; $\square, 391 \mathrm{~K} ; 0,386 \mathrm{~K}$.

et al. ${ }^{12}$ that a smaller compound can more easily achieve such a condition supports our suggestion.

Some papers have reported that the diffusion of disperse dyes in polymers such as poly(ethylene terephthalate), ${ }^{12}$ polypropylene, ${ }^{13}$ and polystyrene ${ }^{14}$ is hardly dependent on the initial dye concentration, when it is below $1.5 \times 10^{-4} \mathrm{~mol} \mathrm{~g}^{-1}$ of the polymer. Since the initial probe concentration used here is 


\section{K. HAMAdA et al.}

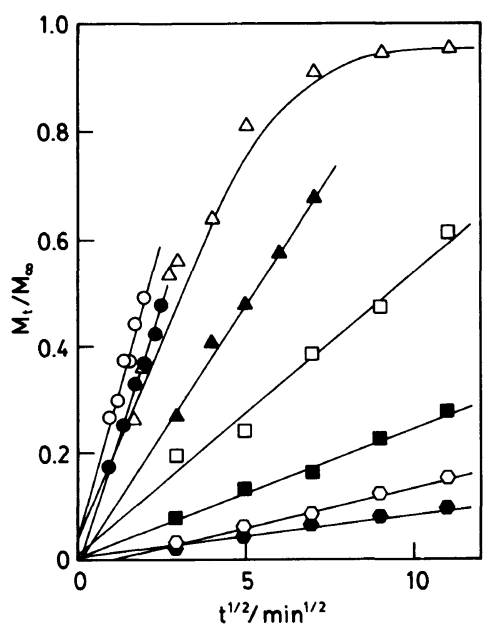

Figure 3. Reduced desorption curves for SPIV in NY11. $\bigcirc, 400 \mathrm{~K}$;, $395 \mathrm{~K} ; \triangle, 391 \mathrm{~K} ; \Delta, 382 \mathrm{~K} ; \square, 372 \mathrm{~K}$; 口, $361 \mathrm{~K} ; \mathrm{O}, 356 \mathrm{~K} ; \bullet, 351 \mathrm{~K}$.

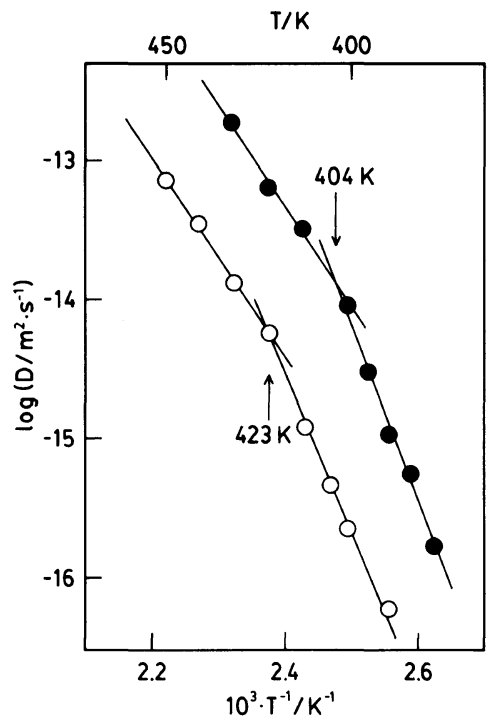

Figure 4. Arrhenius plots of $D$. SPI. $\bigcirc, N Y-6(2 D)$; NY-11.

$(1-5) \times 10^{-5} \mathrm{molg}^{-1}$ of the nylon, the concentration dependence of the diffusion coefficient should be negligible.

Furthermore, the experimental points for SPIV in NY-11 at $391 \mathrm{~K}$ (Figure 3) were compared with the theoretical reduced desorption curve (solid line) calculated from

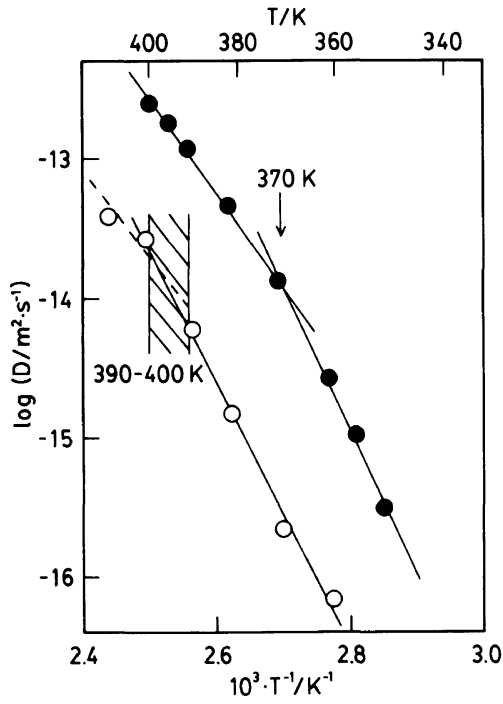

Figure 5. Arrhenius plots of $D$. SPIV. $\bigcirc$, NY-6(2D); , NY-11.

Table I. Crossover points in the Arrhenius plots of the translational and rotational diffusion coefficients

\begin{tabular}{|c|c|c|}
\hline & $T_{\mathrm{n}}^{\mathrm{T}} / \mathrm{K}$ & $T_{\mathrm{n}}^{\mathrm{R}} / \mathrm{K}$ \\
\hline \multicolumn{3}{|l|}{ SPI } \\
\hline$N Y-6(2 D)$ & 423 & 423 \\
\hline NY-11 & 404 & 403 \\
\hline \multicolumn{3}{|l|}{ SPIV } \\
\hline NY-6(2D) & $390-400$ & 390 \\
\hline NY-11 & 370 & 374 \\
\hline
\end{tabular}

McBain's formula (eq 2), ${ }^{15}$ showing fairly good agreement. Here, considering the experimental errors, we calculated the theoretical curve with assumption that $M_{t} / M_{\infty}=0.04$ at $t=0$ and $M_{t} / M_{\infty}=0.95$ at $t=\infty$. This strongly indicates that the diffusion process is within the assumptions of McBain's formula as Masuko et al. pointed out. ${ }^{14}$

$$
\begin{aligned}
\frac{M_{t}}{M_{\infty}}= & 1-\frac{8}{\pi^{2}} \sum_{n=0}^{\infty} \frac{1}{(2 n+1)^{2}} \\
& \times \exp \left\{-\frac{(2 n+1)^{2} \pi^{2} D t}{l^{2}}\right\}
\end{aligned}
$$

The Arrhenius plots of the $D$ values thus 
obtained are shown in Figures 4 and 5. All the plots except that for SPIV in NY-6(2D) had one distinct crossover point $T_{\mathrm{n}}^{\mathrm{T}}$ in the temperature range examined. In the case of SPIV in NY-6(2D), it was difficult to measure the $D$ values at a temperature higher than $400 \mathrm{~K}$, because SPIV easily sublimed in the course of preheating. However, it is worthwhile to point out that $D$ at $T_{\mathrm{n}}^{\mathrm{T}}$ is $1 \times 10^{-14} \mathrm{~m}^{2} \mathrm{~s}^{-1}$ in the other three cases. From this result, we presume that a crossover point for SPIV in NY-6(2D) exists in the temperature range from 390 to $400 \mathrm{~K}$. As shown in Table $\mathrm{I}, T_{\mathrm{n}}{ }^{\mathrm{T}}$ was higher for SPI than for SPIV, indicating that the larger probe molecule changes its mobility at a higher temperature. $T_{\mathrm{n}}^{\mathrm{T}}$ decreased with an increase in the methylene chain length of the nylon. From this, it is thought that the translational mobility increases with increasing methylene chain length of the nylon.

From these Arrhenius plots, the activation energies for translation $E_{\mathrm{a}}^{\mathrm{T}}$ were calculated, and are given in Table II. The $E_{\mathrm{a}}{ }^{\mathrm{T}}$ values were smaller above $T_{\mathrm{n}}^{\mathrm{T}}$ than below it. This fact may be explained by the change of the motional mode of the nylon chains at $T_{\mathrm{n}}^{\mathrm{T}}$, i.e., the increased mobility of the nylon chains makes it easier for the probe molecules to diffuse translationally. In addition, $D$ for Brownian translational diffusion is expressed by the Stokes-Einstein equation.

$$
D=\frac{k T}{6 \pi \eta a_{\mathrm{D}}}
$$

Where $\eta$ is the viscosity of the medium, $a_{\mathrm{D}}$ is the molecular hydrodynamic radius for diffusion, and $k$ is the Boltzmann's constant. The temperature dependence of $D$ includes that of $\eta$, so that the difference between $E_{\mathrm{a}}^{\mathrm{T}}$ above and below $T_{\mathrm{n}}^{\mathrm{T}}$ reflects the change of the temperature dependence of $\eta$ caused by the motional change of the polymer chains.

$E_{\mathrm{a}}^{\mathrm{T}}$ was independent of the methylene chain length of the nylons, within the rather large experimental error, but it was larger for SPI than for SPIV in the "low" temperature region

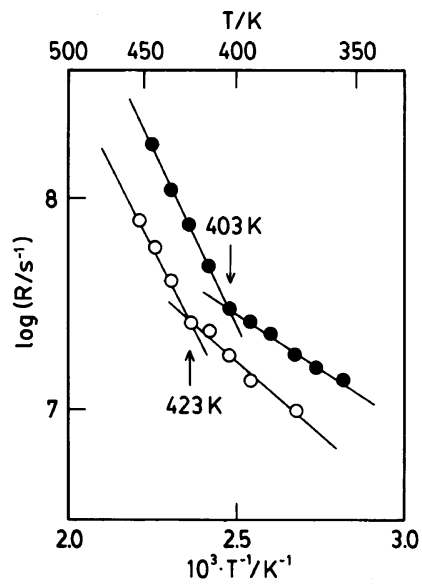

Figure 6. Arrhenius plots of $R$. SPI. $\bigcirc$, NY-6(2D); NY-11.

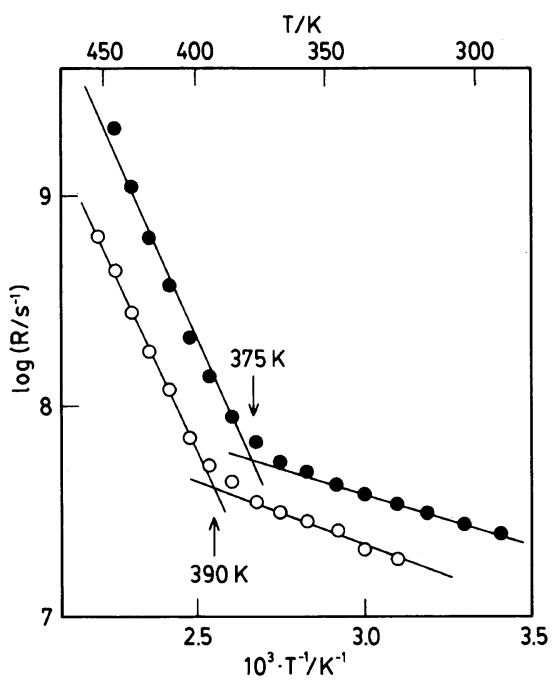

Figure 7. Arrhenius plots of $R$. SPIV. $\bigcirc$, NY-6(2D); , NY-11.

below $T_{\mathrm{n}}^{\mathrm{T}}$, suggesting that the molar volumes of the spin probes affect the translational diffusion below $T_{\mathrm{n}}^{\mathrm{T}}$. In the "high" temperature region, however, the influence of the molar volumes was small: both the spin probes probably monitor the segmental motions of the nylon chains, and hence the activation energies mainly include those for the segmental motions.

On the other hand, the rotational diffusion 
coefficients $R$ were calculated from the rotational correlation times $\tau_{\mathbf{R}}$ obtained in our previous papers, ${ }^{3,6}$ assuming a simple isotropic Brownian reorientation process. ${ }^{1}$

$$
R=\frac{1}{6 \tau_{\mathrm{R}}}
$$

The Arrhenius plots of $R$ are shown in Figures 6 and 7. These plots also had one distinct crossover point in the temperature region examined for the translational diffusion of the probe molecules. The crossover point is designated as $T_{\mathrm{n}} \mathrm{R}$. (It was designated as $T_{\mathrm{n}}$ in our previous paper. ${ }^{3,6}$ ) For both the spin probes, another crossover point $T_{\mathrm{n}}{ }^{\prime}$ could be defined in the temperature region below that shown in Figures 6 and 7.,6 In the case of SPI, rotation around the single bond linking the probe moiety with the NH group probably occurs; the temperature at which this singlebond rotation takes place is assumed to correspond to $T_{\mathrm{n}}{ }^{\prime}$ for SPI. ${ }^{3}$ SPIV has no single bonds around which to rotate, and consequently $T_{\mathrm{n}}{ }^{\prime}$ for SPIV is believed to be the temperature where the rotation of the whole probe molecule occurs without translation. ${ }^{6}$ On the other hand, $T_{\mathrm{n}}{ }^{\mathrm{R}}$ is probably the temperature at which the probe molecule starts to rotate together with translational diffusion., ${ }^{3,6}$ The $T_{\mathrm{n}}{ }^{\mathrm{R}}$ values thus obtained were quite con- sistent with the $T_{\mathrm{n}}{ }^{\mathrm{T}}$ values (Table I), suggesting that the motional changes of the nylon chains influence not only the translational motions but also the rotation. $R$ for a simple isotropic Brownian reorientation process is also related to $\eta$ as shown in eq 5 .

$$
R=\frac{k T}{8 \pi \eta a_{\mathrm{R}}{ }^{3}}
$$

where $a_{\mathrm{R}}$ is the molecular hydrodynamic radius for rotation. The experimental agreement between $T_{\mathrm{n}}{ }^{\mathrm{T}}$ and $T_{\mathrm{n}}{ }^{\mathrm{R}}$ suggests that the change of the temperature dependence of $\eta$ at the crossover point is probably reflected by both the translational and rotational motions of the spin probes. In the subsequent section, therefore, both $T_{\mathrm{n}}{ }^{\mathrm{T}}$ and $T_{\mathrm{n}}{ }^{\mathrm{R}}$ are designated as $T_{\mathrm{n}}$.

The activation energies for rotation $E_{\mathrm{a}}{ }^{\mathrm{R}}$ were determined from Figures 6 and 7 (Table II). (The temperature range designated as "low" in the table is given as "medium" in our previous papers. $^{3,6}$ ) The $E_{\mathrm{a}}{ }^{\mathrm{R}}$ values were larger above $T_{\mathrm{n}}$ than below it; this result is the opposite to that for translation. This may be explained as follows. The ESR technique used can detect rotational motion with a correlation time ranged at $10^{-11}-10^{-7} \mathrm{~s}$, but gives no information about motion with a rotational correlation time greater than $10^{-7}$ or less than $10^{-11} \mathrm{~s}$. As the $E_{\mathrm{a}}^{\mathrm{T}}$ values were smaller above

\begin{tabular}{|c|c|c|c|c|c|}
\hline & \multicolumn{2}{|c|}{$E_{\mathrm{a}}^{\mathrm{T}} / \mathrm{kJ} \mathrm{mol}^{-1}$} & \multicolumn{2}{|c|}{$E_{\mathrm{a}}^{\mathrm{R}} / \mathrm{kJ} \mathrm{mol}^{-1}$} & \multirow{2}{*}{$\frac{\beta\left(E_{\mathrm{a}}^{\mathrm{T}} / E_{\mathrm{a}}^{\mathrm{R}}\right)}{\text { High }}$} \\
\hline & Low & High & Low & High & \\
\hline \multicolumn{6}{|l|}{ SPI } \\
\hline NY-6(2D) & $213 \pm 9$ & $138 \pm 6$ & $27 \pm 2$ & $59 \pm 4$ & $2.3 \pm 0.3$ \\
\hline NY-11 & $240 \pm 20$ & $141 \pm 10$ & $19.6 \pm 1.2$ & $63.2 \pm 1.9$ & $2.2 \pm 0.2$ \\
\hline \multicolumn{6}{|l|}{ SPIV } \\
\hline NY-6(2D) & $183 \pm 8$ & - & $11.1 \pm 0.8$ & $64 \pm 2$ & - \\
\hline NY-11 & $195 \pm 16$ & $131 \pm 6$ & $8.92 \pm 0.13$ & $69 \pm 4$ & $1.9 \pm 0.2$ \\
\hline Polyethylene $^{\mathrm{a}}$ & - & $5 \overline{5}$ & 二 & $4 \overline{5}$ & 1.2 \\
\hline Natural rubber ${ }^{b}$ & - & $54 \pm 3$ & $11.3 \pm 1.7$ & $27 \pm 3$ & $2.0 \pm 0.3$ \\
\hline Butyl rubber ${ }^{b}$ & - & $51 \pm 3$ & $7.9 \pm 1.7$ & $33 \pm 3$ & $1.5 \pm 0.2$ \\
\hline
\end{tabular}

Table II. Activation energies for translation and rotation

a $C f$. ref 7 .

b $C f$. ref 9. 
(a) Temperature $<T_{n}$

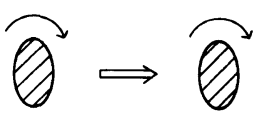

(b) Temperature $>T_{n}$

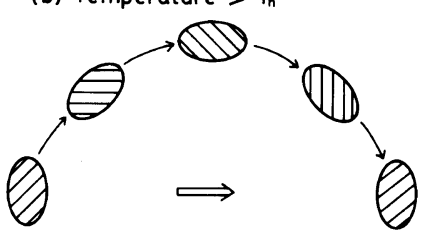

Figure 8. A schematic model of translational and rotational diffusion.

$T_{\mathrm{n}}$ than below, the probe molecules diffuse translationally more easily above $T_{\mathrm{n}}$ than below, i.e., the mode of the translational diffusion changes at $T_{\mathrm{n}}$ as shown in Figure 8. Furthermore, the $E_{\mathrm{a}}^{\mathrm{R}}$ values became larger beyond $T_{\mathrm{n}}$ and closer to the $E_{\mathrm{a}}^{\mathrm{T}}$ values in the "high" temperature region, suggesting that $E_{\mathrm{a}}^{\mathbf{R}}$ in the "high" temperature region includes a part of the activation energy for translation in addition to that for rotation. Accordingly, the ESR information includes not only the rotational diffusion but also the translational diffusion of the spin probes in the "high" temperature region above $T_{n}$. From the above results, we can conclude that at $T_{\mathrm{n}}$ the probe molecules begin a motion in which their translational jumping becomes coupled with the jumping of the polymer seg- ments.

The activation energies for rotation have been compared with the activation energies for relaxation processes in polymers. ${ }^{16-19}$ For nylon 6,10 , the rotational mobility of the spin probes corresponds to the motions of the short polymer segments ( $\gamma$ relaxation) in the slow rotation region. ${ }^{17}$ According to the results of other polymers, ${ }^{18,19}$ the rotational mobility of the spin probes in the rapid rotation region correspond to the $\alpha+\beta$ relaxation. Thus the $E_{\mathrm{a}}^{\mathrm{R}}$ values are smaller than the activation energies for $\alpha$ relaxation. However, the $E_{\mathrm{a}}^{\mathrm{T}}$ values were larger than $E_{\mathrm{a}}{ }^{\mathrm{R}}$ values even in the rapid rotation region (the "high" temperature region), suggesting that the activation energies for translation reflect those for $\alpha$ relaxation rather than for $\beta$ relaxation. From this, the total motions of the probe molecules monitor those of the polymer chains.

The results obtained here were compared with those obtained for other polymers. ${ }^{7,9}$ The values in the "high" temperature region are discussed. The $E_{\mathrm{a}}^{\mathrm{T}}$ values were much larger for the nylons than for polyethylene, natural rubber, and butyl rubber. The $E_{\mathrm{a}}{ }^{\mathrm{R}}$ values behaved in the same manner. These results may be explained as follows. The nylons have amide groups, and their molecular motions are restricted owing to the hydrogen bonds between the polymer chains, so that the translational and rotational diffusion of the small molecules

Table III. Preexponential factor in the "high" temperature region

\begin{tabular}{|c|c|c|c|}
\hline & $D_{0} / \mathrm{m}^{2} \mathrm{~s}^{-1}$ & $R_{0} / \mathrm{s}^{-1}$ & $\log \alpha\left(\log \left(D_{0} / R_{0}{ }^{\beta}\right)\right)$ \\
\hline \multicolumn{4}{|l|}{ SPI } \\
\hline NY-6(2D) & $7.1 \times 10^{2}$ & $4.8 \times 10^{14}$ & -30.9 \\
\hline$N Y-11$ & $2.1 \times 10^{4}$ & $4.6 \times 10^{15}$ & -30.1 \\
\hline \multicolumn{4}{|l|}{ SPIV } \\
\hline NY-6(2D) & - & $1.4 \times 10^{16}$ & - \\
\hline NY-11 & $3.3 \times 10^{4}$ & $2.2 \times 10^{17}$ & -28.4 \\
\hline Polyethylene $^{\mathrm{a}}$ & $1.0 \times 10^{-3}$ & $1.2 \times 10^{16}$ & -22.3 \\
\hline Natural rubber ${ }^{b}$ & $8 \times 10^{-3}$ & $3.1 \times 10^{13}$ & -29.1 \\
\hline Butyl rubber ${ }^{\mathbf{b}}$ & $2.3 \times 10^{-5}$ & $7.2 \times 10^{13}$ & -18.7 \\
\hline
\end{tabular}

a $C f$. ref 7 .

b $C f$. ref 9 . 
is probably restricted.

To elucidate the mechanism of the diffusion, the use of an empirical equation (eq 6) described by Vasserman et al..$^{9}$ is convenient.

$$
D=\alpha R^{\beta}
$$

where $\beta=E_{\mathrm{a}}{ }^{\mathrm{T}} / E_{\mathrm{a}}{ }^{\mathrm{R}}$ and $\alpha=D_{0} / R_{0}{ }^{\beta} . D_{0}$ and $R_{0}$ are the preexponential factors for translation and rotation, respectively (Table III). $\alpha$ and $\beta$ are probably classified into two groups. For NY-6(2D), NY-11, and natural rubber, $\log \alpha=28-31$ and $\beta=1.9-2.3$, while for polyethylene and butyl rubber, $\log \alpha=18-23$ and $\beta=1.0-1.5$. This classification is the same as that reported by Vasserman et al. ${ }^{9}$ From the above results, we can conclude that the diffusion mechanism of the spin probe in the nylon is similar to that in natural rubber.

Furthermore, the value $n=\tau_{\mathrm{T}} / \tau_{\mathrm{R}}$ (where $\left.\tau_{\mathrm{T}}=x^{2} / 6 \mathrm{D}\right)$ was calculated for SPIV in NY-11. $n$ shows how many times a radical changes its orientation over the translation distance of $x=$ $0.5 \mathrm{~nm}$ which is approximately equal to its diameter. Figure 9 shows the plot of $n$ as a function of $R$. In the figure, the results for SPIV in natural rubber (solid line) and in butyl rubber (dashed line) reported in the literature ${ }^{9}$ are shown together with those in NY-11 (open

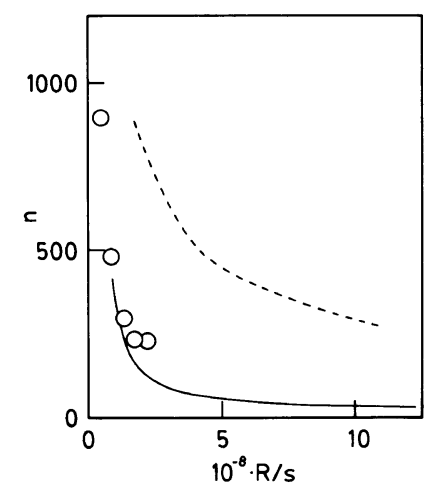

Figure 9. Relation between $n$ and $R$. SPIV. $\bigcirc$, NH-11; $\longrightarrow$, natural rubber; ----, butyl rubber. circle). $n$ was much larger than unity, indicating that rotation of a spin probe in a polymer is a much faster process than its translation, as concluded by Vasserman et al. ${ }^{9}$ The values for NY-11 were located on the curve for natural rubber. This also suggests that the diffusion mechanism of SPIV in the nylon is similar to that in natural rubber. It is thus concluded that $n$ is very dependent on the state of polymer.

\section{REFERENCES}

1. R. McGregor, T. Iijima, T. Sakai, R. D. Gilbert, and K. Hamada, J. Membrane Sci., 18, 129 (1984).

2. K. Hamada, T. Iijima, and R. McGregor, Polym. J., 17, 1245 (1985).

3. K. Hamada, T. Iijima, and R. McGregor, Macromolecules, 19, 1443 (1986).

4. K. Hamada, T. Iijima, and R. McGregor, J. Polym. Sci., Polym. Phys. Ed., in press.

5. K. Hamada, T. Iijima, and R. McGregor, Macromolecules, 20, 215 (1987).

6. K. Hamada, T. Iijima, and R. McGregor, Polym. J., 19, 709 (1987).

7. V. S. Stryukov, Dokl. Acad. Nauk. SSSR, 179, 641 (1968).

8. A. L. Kovarskii, A. M. Vasserman, and A. L. Buchachenko, Dokl. Akad. Nauk. SSSR, 201, 1385 (1971).

9. A. M. Vasserman, I. I. Barashkova, L. L. Yasina, and V. S. Pudov, Vysokomol. Soyedin., Ser. A, 19, 2083 (1977).

10. I. I. Barashkova and A. M. Vasserman, Vysokomol. Soyedin., Ser. A, 22, 2540 (1980).

11. A. P. Griva and B. E. Krisyuk, Zh. Fiz. Khim., 59, 1624 (1985).

12. I. Ito, S. Okajima, and F. Shibata, J. Appl. Polym. Sci., 14, 551 (1970).

13. S. Okajima, N. Sato, and M. Tasaka, J. Appl. Polym. Sci., 14, 1563 (1970).

14. T. Masuko, M. Sato, and M. Karasawa, J. Appl. Polym. Sci., 22, 1431 (1978).

15. J. W. McBain, Z. Phys. Chem., 68, 471 (1909).

16. A. L. Kovarskii, J. Placek, and F. Szöcs, Polymer, 19, 1137 (1978).

17. P. Törmälä, J. Macromol. Sci., Rev. Macromol. Chem., C17, 297 (1979).

18. F. Lembicz, Makromol. Chem., 186, 665 (1985).

19. F. Lembicz and R. Ukielski, Makromol. Chem., 186, 1679 (1985). 